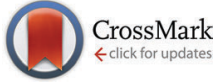

Cite this: Phys. Chem. Chem. Phys., 2015, 17, 25905

Received 15th March 2015, Accepted 26th May 2015

DOI: $10.1039 / c 5$ cp01518a

www.rsc.org/pccp

\section{Can far-IR action spectroscopy combined with BOMD simulations be conformation selective? $\dagger$}

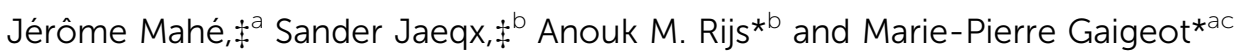

The combination of conformation selective far-IR/UV double resonance spectroscopy with BornOppenheimer molecular dynamics (BOMD) simulations is presented here for the structural characterization of the Ac-Phe-Pro- $\mathrm{NH}_{2}$ peptide in the far-infrared spectral domain, i.e. for radiation below $800 \mathrm{~cm}^{-1}$. Two conformers have been shown to be present in the experiment, namely a conformer with a $\gamma$-turn fold (C7 interaction) and a $\beta$-turn fold (C10 interaction). The combined experimental and theoretical work presented here aims to provide spectral features typical of each conformer in this far-IR domain. The simulated BOMD far-IR spectra agree well with the experimental spectra and allow direct assignment of the observed bands. These assignments show that the $400-550 \mathrm{~cm}^{-1}$ spectral domain is conformer selective, allowing us to distinguish the $\mathrm{H}$-bond signature of the $\gamma$-turn from the $\beta$-turn.
\end{abstract}

\section{Introduction}

Understanding the forces that govern the complex protein folding process is one of the holy grails in modern biophysical science. ${ }^{1}$ One way to obtain insights into this process is to study the folding propensities of small isolated peptides by observing the emergence of secondary structures through intramolecular hydrogen bonding. ${ }^{2}$ Conformation-selective mid-IR spectroscopy in combination with harmonic density functional theory (DFT) calculations has proven to be a powerful tool to this end, and is nowadays applied on a routine-basis. ${ }^{3-6}$ The far-IR region (radiation $<800 \mathrm{~cm}^{-1}$ ) has often been ignored, due to the possible deficiencies of theoretical tools needed to interpret the experimental spectra in this domain. ${ }^{7-9}$ This far-IR regime not only complements the mid-IR one, but also yields information that is not accessible in the mid-IR region. The mid-IR region mostly probes localized structural information, due to the localized character of the vibrations found in this region, such as the structurally diagnostic amide A ( $\mathrm{NH}$ stretch vibration), amide I $(\mathrm{C}=\mathrm{O}$ stretch vibration $)$ and amide II ( $\mathrm{NH}$ in-planebending vibration) modes of peptides. On the other hand, the far-IR region is characterized by large-scale delocalized vibrations. These vibrations are expected to contain detailed structural

\footnotetext{
${ }^{a}$ LAMBE CNRS UMR8587, Université d'Evry val d'Essonne, Blvd F. Mitterrand, Bât Maupertuis, 91025 Evry, France

${ }^{b}$ Radboud University, Institute for Molecules and Materials, FELIX Laboratory, Toernooiveld 7c, 6525 ED Nijmegen, The Netherlands.E-mail: a.rijs@science.ru.nl ${ }^{c}$ Institut Universitaire de France, 103 Blvd St Michel, 75005 Paris, France.

E-mail: mgaigeot@univ-evry.fr

$\dagger$ Electronic supplementary information (ESI) available. See DOI: 10.1039/ c5cp01518a

\$ J.M. and S.J. have equally contributed to the work.
}

information on the overall structure and are therefore directly diagnostic to various backbone conformations. ${ }^{10-12}$ Since these vibrations often extend over a large part of the peptide backbone, such delocalized modes are also expected to be important for the dynamical and flexible nature of proteins. ${ }^{13}$ Additionally, intrinsic hydrogen bond vibrations can be directly probed in the far-IR region, ${ }^{14,15}$ as previously shown for condensed phase systems using low-frequency FTIR spectroscopy ${ }^{16,17}$ In contrast, the mid-IR region can indirectly probe hydrogen bonds through frequency shifts of the amide vibrations. One more advantage of far-IR probing is the possibility to probe larger and more complex molecules. For such molecular systems, the mid-IR region is often spectrally congested due to many overlapping amide bands and thereby limiting the conformational assignment to families rather than to one specific conformation. ${ }^{4,18,19}$ In these cases the far-IR spectra often still show resolved absorption bands. This is a consequence of the bandwidth of the free electron laser used in these experiments, which is proportional to the output photon energy. ${ }^{20}$

Synergy between experiments and theoretical calculations is essential to obtain structural information from these lowfrequency motions. Static harmonic DFT calculations are known to be insufficient to achieve this task. In a previously published paper, ${ }^{21}$ we have shown that Born-Oppenheimer molecular dynamics (BOMD) is able to reproduce the far-IR absorption spectra of gas phase peptides, and can therefore be employed to obtain structural information from the far-IR absorption region of peptides. This combination is able to distinguish between subtle differences in peptide conformations, superior to static DFT calculations in combination with mid-IR spectroscopy. For example, far-IR spectroscopy coupled with BOMD simulations could differentiate the axial and equatorial 
forms of the $\gamma$-turn interaction in Ac-Phe-Gly- $\mathrm{NH}_{2}$, which was not possible with mid-IR spectroscopy and static (harmonic) DFT calculations. ${ }^{22,23}$ As will be described in the present paper, BOMD simulations take the anharmonic character of the delocalized vibrations directly into account, providing reliable spectroscopic predictions, vibrational assignments and structural interpretations. The combination between experiment and theory provides direct insight into the nature of the low frequency motions.

Apart from our developed far-IR set-up for the investigation of neutral gas phase peptides using the FELIX free electron laser, ${ }^{21}$ such spectroscopy has been developed in the Havenith group with investigations specifically focused on the probing of the solvation of biomolecules, see for instance ref. 24-26. In the groups of Plusquellic and Markelz, terahertz studies are performed on condensed phase biological systems, from simple amino acids to complete proteins. ${ }^{10,14}$ Far-IR spectra of tagged gas phase ionic clusters have also been obtained by the Asmis group in Berlin, see their review. ${ }^{27}$ Protonated clusters and ionic clusters of atmospherical interest have mainly been investigated with this technique. Lastly, far-IR studies are employed to study the structural properties of metal clusters. ${ }^{28}$

As yet, the far-IR part of the absorption spectrum of gas phase peptides is in many aspects an uncharted territory. To identify the functional vibrations and distinguish them from other bands in the spectrum demands a large understanding of the low frequency modes. We have started such mapping in ref. 21 by combining far-IR experiments and BOMD simulations, and we continue this approach here with Ac-Phe-Pro- $\mathrm{NH}_{2}$ to assess the conformation selectivity capabilities of our approach. Previous experiments on Ac-Phe-Pro- $\mathrm{NH}_{2}$ performed in the 3 micron region (probing the $\mathrm{NH}$ stretch vibrations) by Mons et al. ${ }^{29}$ have shown that the backbone of this peptide can fold either via a $\beta$-turn (the backbone adopts a C10 interaction, with an unusual cis conformation for the Phe-Pro peptide bond) or a $\gamma$-turn (the backbone exhibits a C5 and C7 interaction). These two conformations co-exist in the molecular beam expansion experiments. Choosing this specific peptide allows us to directly observe the difference between backbone folding, here between the C10 and $\mathrm{C} 7$ interactions, in far-IR patterns. In that respect, going from Ac-Phe-Gly- $\mathrm{NH}_{2}$ and Ac-Phe-Ala- $\mathrm{NH}_{2}$ (previous work, ${ }^{21}$ axial and equatorial $\mathrm{C} 7 \gamma$-turns), to Ac-Phe-Pro- $\mathrm{NH}_{2}$ (present work, $\mathrm{C} 7 / \mathrm{C} 10 \mathrm{H}$-bond folding), provides the opportunity to probe different structural motifs whose signatures are identified through far-IR and BOMD vibrational spectroscopy.

\section{Methods}

\subsection{Experimental details}

Ac-Phe-Pro- $\mathrm{NH}_{2}$ (95\% purity) was purchased from Genecust (Dudelange, Luxembourg) and used without further purification. Here, the experimental set-up is briefly described. A complete description of the set-up is described elsewhere. ${ }^{30,31}$ The sample was mixed with graphite powder and applied on a solid graphite bar. A pulsed YAG laser operating at $1064 \mathrm{~nm}$
(Polaris Pulsed Nd:YAG Laser, NewWave Research) with a pulse energy of about $1.5 \mathrm{~mJ}$ was used to desorb the sample molecules from the graphite substrate as intact neutral molecules. The gas-phase molecules are entrained in a supersonic molecular beam of argon, produced by a pulsed valve (Jordan) and a backing pressure of 3 bar of argon. In the molecular beam, the peptide molecules are cooled towards their rotational and vibrational ground state. ${ }^{32}$ The molecular beam travels through a skimmer of about $10 \mathrm{~cm}$ downstream to enter a differentially pumped chamber equiped with a reflectron time-of-flight mass spectrometer. Here, the molecules interact with a UV beam produced by a pulsed Nd:YAG laser (either Innolas GmbH Spitlight 1200 or Quanta-Ray Lab Series) coupled to a frequency doubled dye laser (Radiant Dyes NarrowScan, laser dye: coumarin 153). The UV laser was operated at $10 \mathrm{~Hz}$ with typical pulse energies of 1-2 mJ. The molecules are 2-photon ionized via a one color $(1+1)$ REMPI scheme. Conformation selection is achieved by fixing the UV laser at a specific $S_{1} \leftarrow S_{0}$ transition. Since different conformations have a slightly different electronically excited state energy, they will therefore appear as different peaks in the UV excitation spectrum. The generated ions are accelerated into the time-of-flight tube, and reflected into the detector.

For the IR-UV double resonance spectra, the IR and UV beams were spatially overlapped, but the IR pulse precedes the UV pulse by $\sim 200 \mathrm{~ns}$. The IR radiation is produced by the Free Electron Laser for Infrared eXperiments (FELIX) located in the FELIX laboratory at the Radboud University. ${ }^{20}$ The frequency of the UV probe pulse is fixed on a transition producing a constant ion signal. For the $\gamma$-turn conformation the UV laser was set at $37435.5 \mathrm{~cm}^{-1}$, while at $37409 \mathrm{~cm}^{-1}$ for the $\beta$-turn conformer (see Fig. S1 of the ESI $\dagger$ ). Whenever the IR hole-burn laser excites a transition that shares the same ground state as the probe laser (and thus is the same conformation), a dip in the ion signal is observed since the ground state is depleted by the IR laser. An IR absorption spectrum of a single conformer can thus be constructed by taking the logarithm of the ion signal without the IR pulse divided by the ion signal with IR pulse. To correct for long-term UV power drifts and changing source conditions, alternating IR-on and IR-off signals are measured by operating the IR laser at $5 \mathrm{~Hz}$ and the UV laser at $10 \mathrm{~Hz}$. Since the experiments are performed over a very wide frequency range, the IR laser intensity needs to be corrected for the photon energy. A photon flux resulting in a $1 \mathrm{~mJ}$ pulse energy at $1000 \mathrm{~cm}^{-1}$ is equal to a photon flux resulting in a $0.1 \mathrm{~mJ}$ pulse energy at $100 \mathrm{~cm}^{-1}$, assuming that the laser pulse profile is identical at the two photon energies. Therefore, the observed absorption intensities are multiplied with the photon energy (in wavenumbers) and renormalized to correct for this effect.

\subsection{Theoretical details}

Our theoretical methodology consists of DFT-based molecular dynamics simulations, performed within the Born-Oppenheimer (BOMD) framework using the CP2K package. ${ }^{33,34}$ The methods and algorithms employed in the $\mathrm{CP} 2 \mathrm{~K}$ package are described in detail in ref. 33. In our dynamics, the nuclei are treated classically 
and the electrons quantum mechanically within the DFT formalism. Dynamics consist of solving Newton's equations of motion at a finite temperature, with the forces that act on the nuclei derived from the Kohn-Sham energy. In BOMD, the Schrödinger equation for the electronic configuration of the system is solved at each time step of the dynamics. Mixed plane waves and gaussian basis sets are used in CP2K. Only the valence electrons are taken into account and pseudo-potentials of the Goedecker-Tetter-Hutter (GTH) form are used. ${ }^{35-37}$ We use the Becke, Lee, Yang and Parr (BLYP) gradient-corrected functional ${ }^{38,39}$ for the exchange and correlation terms. Dispersion interactions have been included with the Grimme D3 corrections. ${ }^{40}$ Calculations are restricted to the $\Gamma$ point of the Brillouin zone. We employed plane-wave basis sets with a kinetic energy cut-off of 450 Ry and gaussian basis sets of aug-TZV2P type. The cubic box size is of $20 \AA$ length. The kinetic energy cut-off, basis set size and cubic box size have been selected subsequent to energy convergence tests.

The first 3 ps of the trajectory was used for the thermalisation of the system with temperature control through velocity rescaling. Hereafter, pure NVE trajectories were accumulated over 20 ps for the IR spectra calculations and trajectory analyses. Periodic boundary conditions were applied (neutral molecule). The time step in the simulations is 0.4 fs. The temperature of the trajectories was $48 \pm 4 \mathrm{~K}$ and $53 \pm 5 \mathrm{~K}$, respectively, for the $\gamma$-turn and $\beta$-turn conformers.

Within statistical mechanics and linear response theory, ${ }^{41,42}$ an infrared spectrum can be calculated by the Fourier transform of the time correlation function of the fluctuating dipole moment vector of the absorbing molecular system:

$$
I(\omega)=\frac{2 \pi \beta \omega^{2}}{3 c V} \int_{-\infty}^{-\infty} \mathrm{d} t\langle\delta \mathbf{M}(t) \cdot \delta \mathbf{M}(0)\rangle \exp (i \omega t)
$$

where $\beta=1 / k T, T$ is the temperature, $c$ is the speed of light in a vacuum, $V$ is the volume. The angular brackets represent a statistical average of the time correlation function of the dipole vector, where $\delta \mathbf{M}(t)=\mathbf{M}(t)-\langle\mathbf{M}\rangle$ with $\langle\mathbf{M}\rangle$ the time average of $\mathbf{M}(t)$. The calculation in eqn (1) is done in the absence of an applied external field. For the prefactor in eqn (1), we have taken into account an empirical quantum correction factor (multiplying the classical line shape) of the form $\beta \hbar \omega /(1-\exp (-\beta \hbar \omega))$, which was shown by us and others to give accurate results on calculated IR intensities. ${ }^{43-45}$ For more detailed discussions on quantum corrections, see for instance ref. 46-49.

The main advantages of the molecular dynamics (MD) approach in eqn (1) for the calculation of infrared spectra (also called "dynamical spectra" in the remainder of the text) are discussed in detail in our review ${ }^{50}$ and are briefly listed as follows:

- There are no approximations made in eqn (1) apart from the hypothesis of linear response theory, i.e. a small perturbation from the applied external electric field on the absorbing molecular system. Such conditions are always fulfilled in vibrational spectroscopy of interest here. There are no harmonic approximations made, be they on the potential energy surface or on the dipole moment, in contrast to the usual static calculations used in the literature. These approximations are not needed in eqn (1).

- As a consequence, vibrational anharmonicities are naturally taken into account in eqn (1): one thus only needs the knowledge of the time evolution of the dipole moment of the system in order to calculate an anharmonic IR spectrum. This is naturally achieved with molecular dynamics simulations. In fact, the finite temperature dynamics takes place on all accessible parts of the potential energy surface, be they harmonic or anharmonic. The quality of the potential energy surface is entirely contained in the "ab initio" force field used in the dynamics, calculated at the DFT/BLYP + dispersion level in the work presented here. The good to excellent agreement of the absolute (and relative) positions of the different active bands obtained in our theoretical works (see for instance dynamical spectra in the gas phase, ${ }^{51-55}$ in the liquid phase, ${ }^{43,56-59}$ and at solid-liquid and liquid-air interfaces ${ }^{60-62}$ ) is a demonstration that this level of theory is correct.

- Crucial to the present discussion, the calculation of IR spectra with MD is related only to the time-dependent dipole moment of the molecular system, neither requiring any harmonic expansion of the transition dipole moment nor the knowledge of normal modes, in contrast to harmonic calculations. Therefore, if the dipole moments and their fluctuations are accurately calculated along the trajectory, the resulting IR spectrum should be reliable. The vibrations therefore do not directly rely on the curvature of the potential energy surface at the minima on the PES (i.e. normal modes and derivatives using these normal modes used in static DFT calculations) but rather on the time evolution of the electric dipole moment of the molecular system, which is governed by the conformational dynamics at the finite temperature of the simulation. As a consequence, dynamical anharmonic spectra from eqn (1) and harmonic spectra rely on strictly different properties, and presumably require different levels of accuracy for the evaluation of these properties.

- Eqn (1) gives the whole infrared spectrum of a molecular system in one single calculation, i.e. the band positions, the band intensities and the band shapes, through the Fourier transform of a time correlation function. There are no approximations applied, in particular the shape and broadening of the vibrational bands result from the underlying dynamics and mode-couplings of the system at a given temperature.

No scaling factors of any kind are applied to the vibrations extracted from the dynamics. The sampling of vibrational anharmonicities, i.e. potential energy surface, dipole anharmonicities, mode couplings, anharmonic modes, is included in our simulations, by construction, and the application of a scaling factor to the band positions is therefore unnecessary. As reviewed in previous papers, excellent agreements between dynamical spectra and IR-MPD, IR-PD and IR-UV ion dip experiments have been achieved. Any remaining discrepancies between dynamical and experiment spectra should mainly be due to the choice of the DFT/BLYP + dispersion functional as DFT-based dynamics are only as good as the functional itself allows.

The length of the trajectory is related to the vibrational domain to be sampled. One has to keep in mind that the 
time-length has to be commensurate with the investigated vibrational motions. Hence, trajectories at around 5 ps are just enough in order to sample stretching motions in the high frequency domain of $3000-4000 \mathrm{~cm}^{-1}$, provided that several trajectories starting from different initial conformations (structure and/or velocities) are accumulated and averaged for the final IR dynamical spectrum. In the mid-IR domain, trajectories of at least $10 \mathrm{ps}$ each are needed in order to sample the slower stretching and bending motions of the $1000-2000 \mathrm{~cm}^{-1}$ domain. In the far-IR below $1000 \mathrm{~cm}^{-1}$ of interest to the present work, longer trajectories are needed in order to properly sample the much slower motions typical of that domain, i.e. torsional motions and possibly opening/closure of structures, typical of peptide chains. In Fig. 1, we have reported the dynamical IR spectrum of the $\gamma$-turn conformer calculated at each 5 ps of trajectory over $20 \mathrm{ps}$, in the critical $100-400 \mathrm{~cm}^{-1}$ lower frequency part. One can see that a 20 ps trajectory already allows an excellent convergence of the dynamical IR spectrum (a $100 \mathrm{~cm}^{-1}$ motion is sampled 60 times in that time period).

An accurate calculation of anharmonic infrared spectra is one goal to achieve, the assignment of the active bands into individual atomic displacements or vibrational modes is another one. This issue is essential to the understanding of the underlying molecular, structural and dynamical properties. In molecular dynamics simulations, the interpretation of the infrared active bands into individual atomic displacements traditionally relies on Fourier transforms of time correlation functions based on velocities or on positions. ${ }^{50}$ Because of the intrinsic nature of delocalised and coupled modes in the far-IR spectral range, we have adopted here a strategy of assignments in terms of Fourier transforms of intramolecular coordinate (IC) time correlation functions, named ICDOS in the rest of the paper, following our previous work on far-IR spectra: ${ }^{21}$

$$
I_{\mathrm{ICDOS}}(\omega)=\int_{-\infty}^{\infty}\langle\mathrm{IC}(t) \cdot \operatorname{IC}(0)\rangle \exp (i \omega t) \mathrm{d} t .
$$

Fig. 2 provides a scheme of the PhePro molecule together with the labelling of atoms and definitions of dihedral angles

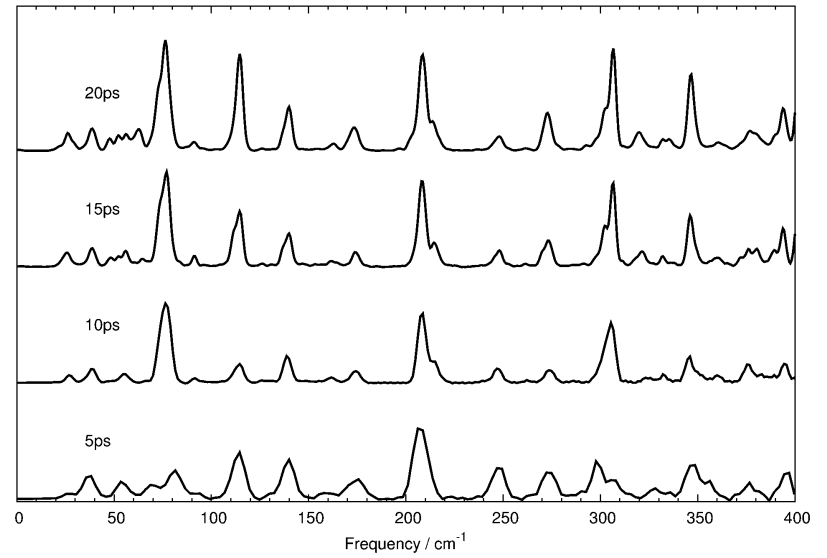

Fig. 1 Dynamical IR spectrum of the $\gamma$-turn conformer in the 100$400 \mathrm{~cm}^{-1}$ domain, calculated at each 5 ps over a 20 ps trajectory, to illustrate the convergence of the spectrum with respect to time-length.

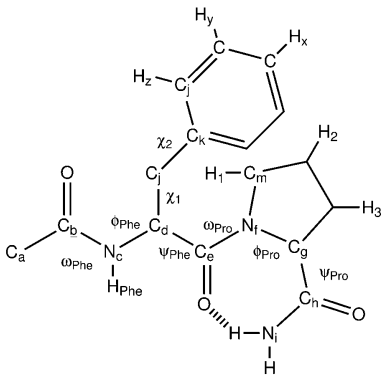

$\gamma$-turn

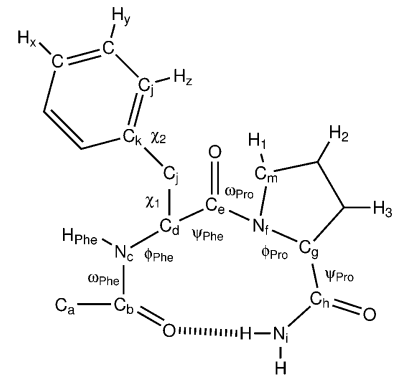

$\beta$-turn
Fig. 2 Scheme of the PhePro molecule with labelling of atom names and dihedral angles $(\phi, \psi, \omega$ and $\chi)$ employed in the text for the assignment of the IR features. The angles are defined as follows, see scheme $2: \phi_{\text {Phe }}=$ $\left(\mathrm{C}_{e}, \mathrm{C}_{\mathrm{d}}, \mathrm{N}_{\mathrm{c}}, \mathrm{C}_{\mathrm{b}}\right), \quad \psi_{\text {Phe }}=\left(\mathrm{N}_{\mathrm{f}}, \mathrm{C}_{\mathrm{e}}, \mathrm{C}_{\mathrm{d}}, \mathrm{N}_{\mathrm{c}}\right), \omega_{\text {Phe }}=\left(\mathrm{O}, \mathrm{C}_{\mathrm{b}}, \mathrm{N}_{\mathrm{c}}, \mathrm{H}_{\mathrm{Phe}}\right), \chi_{1}=$ $\left(N_{c}, C_{d}, C_{j}, C_{k}\right), \chi_{2}=\left(C_{d}, C_{j}, C_{k}, C_{l}\right), \phi_{\text {Pro }}=\left(C_{e}, N_{f}, C_{g}, C_{h}\right), \psi_{\text {Pro }}=\left(N_{f}, C_{g}, C_{h}, N_{i}\right)$, and $\omega_{\text {Pro }}=\left(\mathrm{O}, \mathrm{C}_{\mathrm{e}}, \mathrm{N}_{\mathrm{f}}, \mathrm{C}_{\mathrm{m}}\right)$.

discussed below. As the spectral domain $<1000 \mathrm{~cm}^{-1}$ is related to large amplitude motions, we have chosen intramolecular coordinates such as dihedral angles (from the backbone, see $\phi, \psi$ and $\omega$ in Fig. 2 and Table S2 (ESI $\dagger$ ), from the side chain that carries the phenyl ring, see $\chi_{1}$ and $\chi_{2}$ in Fig. 2), out-of-plane (wagging) motions of $\mathrm{H}$ atoms that belong to the phenyl ring or to the proline residue (labelled dihedral-ring- $\mathrm{H}_{x, y, z}$ and dihedralpro- $\mathrm{H}_{1,2,3}$ in Fig. 2 and 5). We have also calculated the vibrational signature of two coordinates directly related to the $\mathrm{C} 10 / \mathrm{C} 7$ hydrogen bond motion, the $\mathrm{H}$-bond stretching defined as the $\mathrm{NH}_{2} \cdots \mathrm{O}=\mathrm{C}$ distance, and the dihedral angle $\mathrm{H}_{\text {bond }}-\mathrm{N}-\mathrm{C}-\mathrm{C}$ where $\mathrm{H}_{\text {bond }}$ is the $\mathrm{NH}_{2}$ atom hydrogen bonded to the $\mathrm{C}=\mathrm{O}$ group. We have also calculated the vibrational signature of the dihedral angle $\mathrm{H}_{\text {free }}-\mathrm{N}-\mathrm{C}-\mathrm{C}$ where $\mathrm{H}_{\text {free }}$ is the free hydrogen atom of the $\mathrm{NH}_{2}$ group. The signature of the wagging motion of the backbone $\mathrm{N}-\mathrm{H}$ amide group (labelled dihedral- $\mathrm{H}_{\text {Phe }}$ in Fig. 5) has also been calculated.

\section{Results}

\subsection{Conformational assignment}

The REMPI spectrum of Ac-Phe-Pro- $\mathrm{NH}_{2}$ is shown in the ESI $\dagger$ (Fig. S1). The spectrum closely resembles the one previously measured by Mons et al. ${ }^{29}$ We recorded the IR spectra of the major peaks observed in the spectrum and found two different IR spectra. This suggests that two conformations of Ac-Phe-Pro- $\mathrm{NH}_{2}$ are present in our molecular beam expansion experiment. Fig. 3 shows the IR spectra of the $\gamma$-turn (red line) and $\beta$-turn (blue line) recorded from 1850 down to $100 \mathrm{~cm}^{-1}$ with the UV frequency fixed at 37435.5 and $37409 \mathrm{~cm}^{-1}$, respectively. Each IR spectrum shows intense bands throughout the complete IR region, and both spectra show many well-resolved features down to the far-IR region where we observed narrow peaks with a FWHM of about $3 \mathrm{~cm}^{-1}$, limited by the bandwidth of the free electron laser.

The mid-IR region is commonly used to identify peptide structure(s). Here, the amide I region of the $\gamma$-turn conformer is 


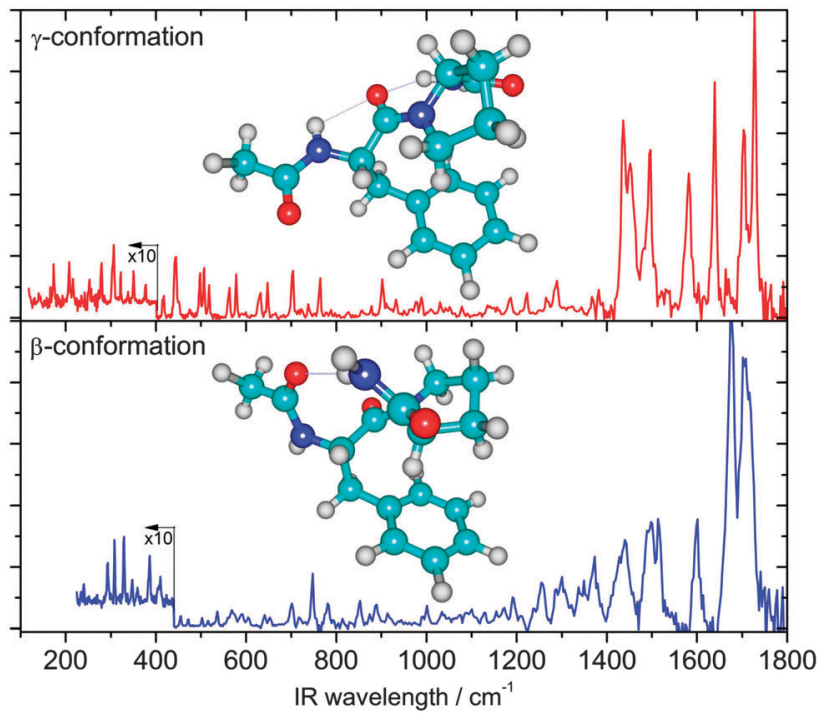

Fig. 3 Recorded IR absorption spectra of the $\gamma$-turn (top) and $\beta$-turn (bottom) in the $100-1850 \mathrm{~cm}^{-1}$ range region. The optimized structures of the assigned structures are shown as well.

composed of three clear peaks between $1780-1610 \mathrm{~cm}^{-1}$, originating from the three backbone $\mathrm{C}=\mathrm{O}$ stretch modes. In contrast, only two peaks are observed for the $\beta$-turn peptide. Here, the three $\mathrm{C}=\mathrm{O}$ stretching modes lie too close to each other to be resolved. The three $\mathrm{C}=\mathrm{O}$ stretch frequencies are calculated to differ by around $22 \mathrm{~cm}^{-1}$. Considering a FWHM of $1-2 \%$ for the FELIX IR source, it is not surprising that these absorption bands are not fully resolved. The medium intense band observed at about $1580 \mathrm{~cm}^{-1}$ ( $\gamma$-turn) and $1600 \mathrm{~cm}^{-1}$ ( $\beta$-turn) results from the $\mathrm{NH}_{2}$ scissor vibration.

The experimental spectrum shown in red in Fig. 3 is readily assigned to the gamma-turn $\left(\beta-\gamma_{\mathrm{L}}\right)$ conformation of PhePro with the phenyl group in the "a orientation". Here, the " $\beta$ " does refer to a C5 interaction which is responsible for the formation of $\beta$-sheets in protein structures. This is the lowest energy structure found in our conformational search and it has the best match in the $1000-1850 \mathrm{~cm}^{-1}$ region, see Fig. S2 (ESI $\dagger$ ). Mons et al. have also assigned the "a phenyl orientation" to the structure based on the Franck-Condon patterns in the REMPI spectrum. ${ }^{29}$

For the spectrum shown in blue in Fig. 3, the assignment is not that straightforward. This spectrum was previously assigned to a type VIa $\beta$-turn conformation with a cis conformation of the Phe-Pro peptide bond by Mons et al. ${ }^{29}$ The "VIa" is used to classify different types of $\beta$-turn conformations. This is also our conclusion from the present work. However, the orientation of the phenyl ring was not discussed in detail in that paper. We therefore performed geometry optimizations of the three different $\mathrm{a}_{2} \mathrm{~g}^{+}$and $\mathrm{g}^{-}$possible orientations of the phenyl group in the $\beta$-turn geometry, and we calculated the associated harmonic IR spectra of the mid-IR region (1000$1800 \mathrm{~cm}^{-1}$, with the B3LYP functional) and for the $3 \mu \mathrm{m}$ region (3000-400 $\mathrm{cm}^{-1}$, with the B97-D functional), including mode dependent scaling factors. ${ }^{63}$ We also performed BOMD simulations (with the BLYP+D3 functional) in order to get the anharmonic far-IR spectra of these three phenyl orientations in the $\beta$-turn geometry of PhePro, see Fig. S3 (ESI $\dagger$ ). The goal is to assign one of these orientations to the experimental spectra. Note that the energies of the $\mathrm{a} \mathrm{g}^{+}$and $\mathrm{g}^{-}$orientation of the phenyl group in the $\beta$-turn optimized structures are $1.22 \mathrm{kcal} \mathrm{mol}^{-1}, 3.85 \mathrm{kcal} \mathrm{mol}^{-1}$ and $3.79 \mathrm{kcal} \mathrm{mol}^{-1}$, respectively, using the B97-D/6-311G+(d,p) level of theory (the $0 \mathrm{kcal} \mathrm{mol}^{-1}$ is assigned to the $\gamma$-turn structure).

These calculations clearly show that the phenyl group is in its "a orientation", see Fig. S3, S4 and Table S1 (ESI $\dagger$ ). For discarding the " $\mathrm{g}^{+}$orientation", the most convincing evidence is found in the $3 \mu \mathrm{m}$ range. As Table S1 (ESI $\dagger$ ) shows, in this orientation the $\mathrm{NH}$ backbone group of Phe interacts with the phenyl ring, causing a strong red shift for the NH stretch vibration. This shift is not observed in the experimental spectrum. The " $\mathrm{g}-$ orientation" can be excluded due to the far too intense
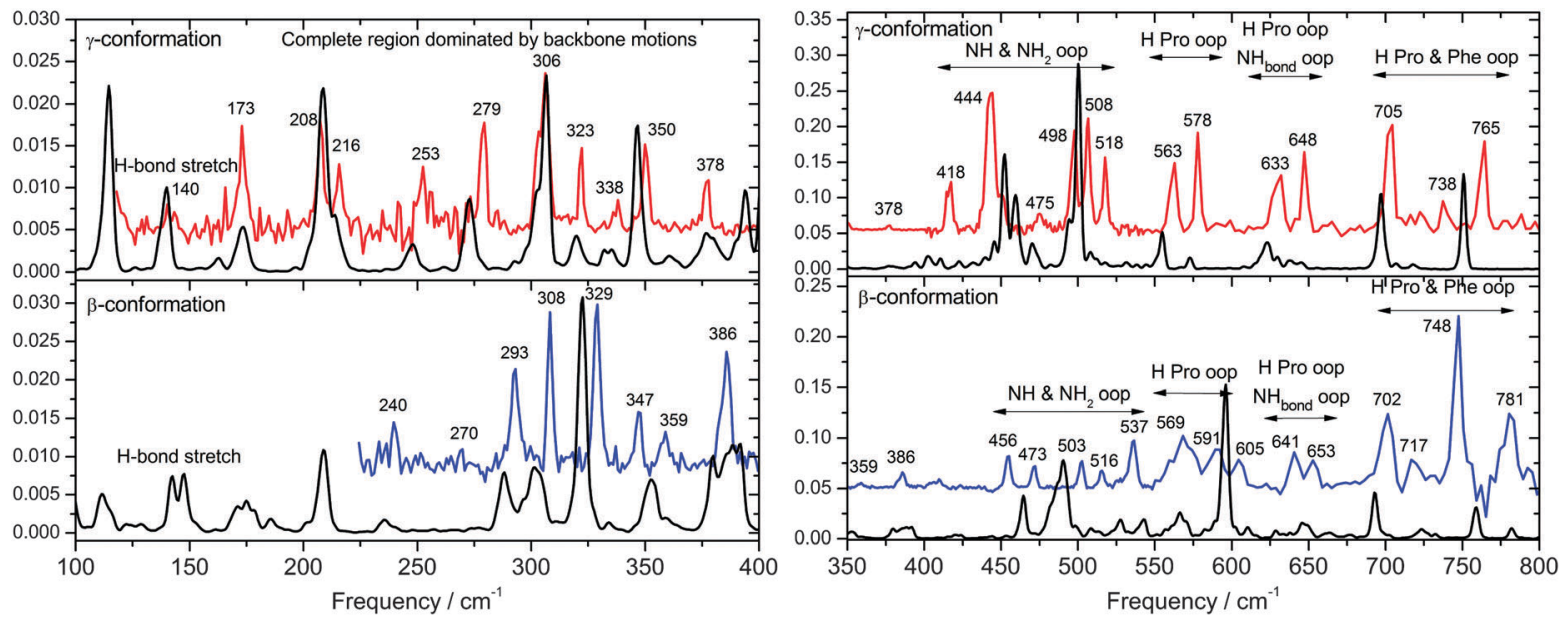

Fig. 4 Comparison between the dynamical BOMD spectra in red and experimental spectra in black in the $100-400 \mathrm{~cm}^{-1}$ domain. The position of the $\mathrm{H}$-bond stretching vibration is indicated as well. Comparison between the dynamical BOMD spectra in red and experimental spectra in black in the 400$800 \mathrm{~cm}^{-1}$ domain. In the experimental spectra various regions are indicated where the assignment between the two conformations is very similar. 
absorption peaks in the far-IR at $408 \mathrm{~cm}^{-1}$ predicted by the BOMD simulations (Fig. S3, ESI $\dagger$ ). The "a orientation" also reproduces the peak patterns in the $1000-1400 \mathrm{~cm}^{-1}$ range (Fig. S4, ESI $\dagger$ ).

The assigned $\gamma$-and $\beta$-turn structures with the "a-orientation" of the phenyl group have been further investigated in our BOMD simulations.

\subsection{BOMD spectra}

Fig. 4 presents the experimental and theoretical IR spectra of the $\gamma$-turn (top) and $\beta$-turn (bottom) conformers of PhePro in the $100-400 \mathrm{~cm}^{-1}$ region and $400-800 \mathrm{~cm}^{-1}$ region respectively. Note that the absorption intensity is lower below $400 \mathrm{~cm}^{-1}$ and the scales in Fig. 4 are adjusted accordingly.

We remind that the dynamical theoretical spectra have not been adjusted in any way (neither band positions, nor bandwidths and shapes). The first observation is that theory and experiment bear remarkable agreement. The theoretical spectra display a number of peaks, positions, band-shapes and intensities that are indeed in good to excellent agreement with the experiments in this anharmonic far-IR frequency range. A few theoretical bands are however too broad in comparison to the experiments and there are also a few bands that either lack intensity or on the contrary carry too much intensity in the theoretical spectra. We will come back to these issues in the discussion. The agreement between experiment and theory is especially remarkable for the $\gamma$-turn conformer. The theoretical spectrum of the $\beta$-turn shows deficiencies with respect to the experiment, mainly in the higher energy region, that are not observed in the theoretical spectrum of the $\gamma$-turn.

One can also observe from the experiments and calculations that the $\beta$-turn and $\gamma$-turn conformers have different signatures in the $100-800 \mathrm{~cm}^{-1}$ domain, thus this region certainly allows us to distinguish both conformers from their IR spectra alone in the far-IR range.

Fig. 5 reports band assignments of the dynamical IR spectra following the method described in Section 2. These assignments are discussed hereafter.

From the experimental and theoretical spectra, one can observe that the $550-800 \mathrm{~cm}^{-1}$ IR spectral region does not appear to be very much conformer selective, as the number of peaks and their positions are very similar between the $\beta$ - and $\gamma$-turn conformers. Making a one-to-one comparison of the position of the peaks between the two conformers in this region, the maximum band-shift that can be observed is roughly $16 \mathrm{~cm}^{-1}$, with an average difference in the peak positions between the two conformers of $\sim 7 \mathrm{~cm}^{-1}$.
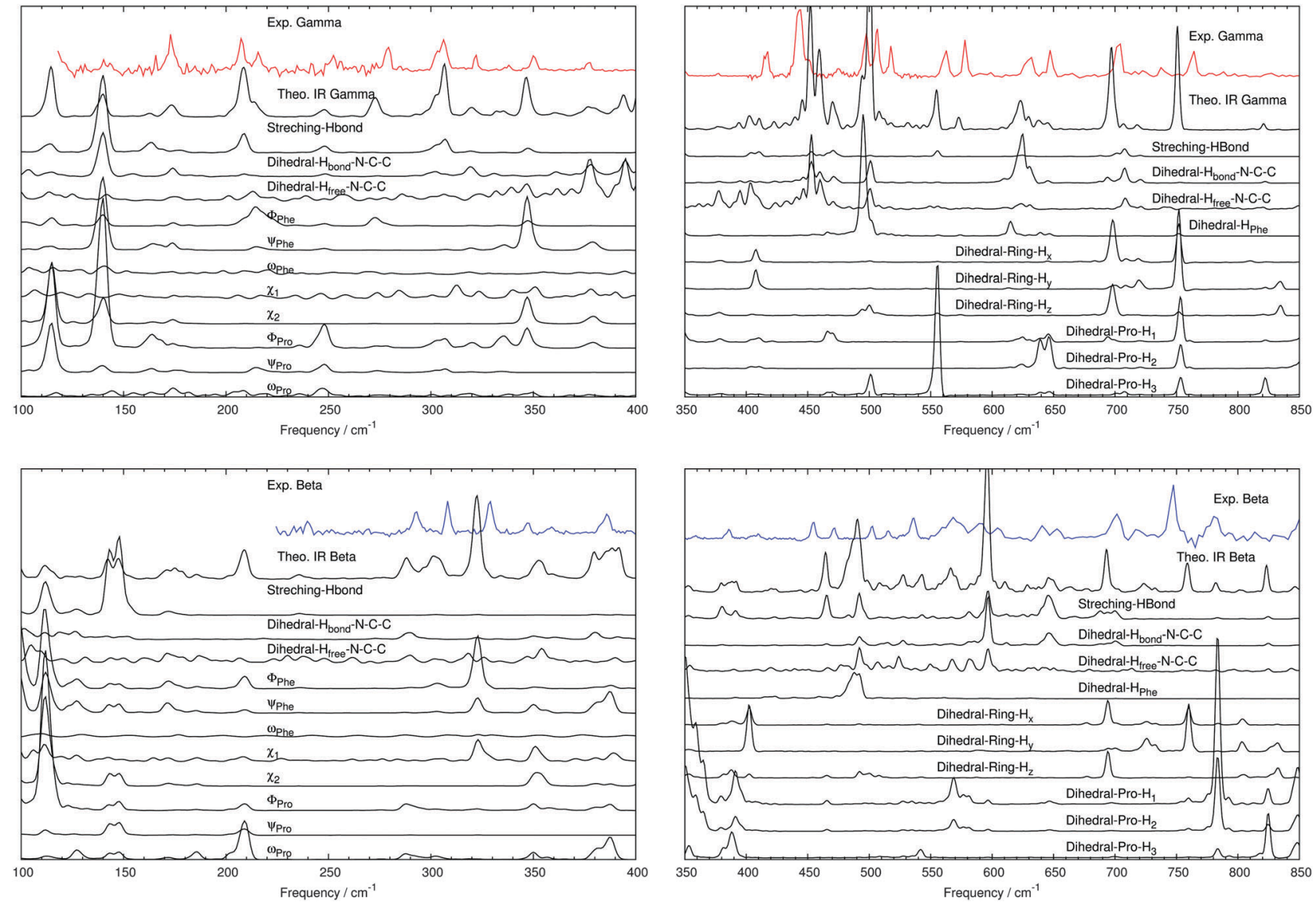

Fig. 5 Decomposition of the vibrational bands of the $\gamma$-turn conformer (top) and the $\beta$-turn conformer (bottom) of PhePro in terms of delocalised motions, using ICDOS, the chosen IC are dihedral motions, $\mathrm{C} 7 \mathrm{H}$-bond motion and $\mathrm{NH}_{2}$ free $\mathrm{H}$ atom dihedral motion. Dihedrals $\phi, \psi, \omega$ and $\chi$ are defined in Fig. 2. See also eqn (2) for the definition of ICDOS. 
That this domain does not appear conformer selective can be very well understood by the band assignments, as we find that the $550-800 \mathrm{~cm}^{-1}$ bands predominantly arise from out-ofplane wagging motions of $\mathrm{H}$ atoms, for both $\beta$ - and $\gamma$-turn conformers of PhePro. These waggings are either due to $\mathrm{H}$ atoms that belong to the phenyl ring or $\mathrm{H}$ atoms belonging to the proline ring residue. As can be seen in the 3D structures in Fig. 3, the two conformers display very similar environments around the Pro and Phe rings, so that one would indeed expect very similar signatures of the $H$ waggings on these two rings, whether they belong to the $\beta$ - or $\gamma$-turn conformers.

The 700-800 $\mathrm{cm}^{-1}$ domain is hence composed of a mixing of out-of-plane wagging motions of $\mathrm{H}$ atoms that belong to the phenyl ring and of $\mathrm{H}$ atoms that belong to the Pro ring. The band located at $700 \mathrm{~cm}^{-1}$ is on the contrary predominantly arising from the out-of-plane waggings of $\mathrm{H}$ atoms that belong to the phenyl ring. One can see that the signatures arising at 705,738 and $765 \mathrm{~cm}^{-1}$ for the $\gamma$ turn $\left(702,717\right.$ and $748 \mathrm{~cm}^{-1}$ for the $\beta$ turn) from $\mathrm{H}$ atoms that belong to the phenyl ring were also present in our previous work about PheAla and PheGly systems. ${ }^{21}$ These features seem to be specific to the aromatic ring. The peaks located at $648 \mathrm{~cm}^{-1}$ for the $\gamma$-turn and 641 and $653 \mathrm{~cm}^{-1}$ for the $\beta$-turn are due to out-of-plane wagging motions of $\mathrm{H}$ atoms of the proline residue.

The C10 ( $\beta$-turn) and C7 ( $\gamma$-turn) hydrogen bond signature seen through the torsional motion of the $\mathrm{H}$-bonded hydrogen atom of $\mathrm{NH}_{2}$ (signature of $\mathrm{H}_{\text {bond }}-\mathrm{N}-\mathrm{C}-\mathrm{C}$ in Fig. 5) also appears in this region. It explains the $633 \mathrm{~cm}^{-1}$ peak for the $\gamma$-turn while this H-bond signature overlaps with the out-of-plane wagging motions of $\mathrm{H}$ atoms of the proline residue for the $\beta$-turn conformer. The two thin peaks at 563 and $578 \mathrm{~cm}^{-1}$ for the $\gamma$-turn conformer and the broader peaks at 569 and $591 \mathrm{~cm}^{-1}$ for the $\beta$-turn are also due to the out-of-plane wagging motions of $\mathrm{H}$ atoms of the proline residue. The participation of the C10 $\mathrm{H}$-bond is also appearing in these bands for the $\beta$-turn conformer. One peak of the doublet lacks intensity in the dynamical spectrum of the $\gamma$-turn conformer, while both peak intensities of the $\beta$-turn conformer are underestimated in the dynamical spectrum.

The 400-550 $\mathrm{cm}^{-1}$ spectral domain appears conformer selective, providing distinct signatures for the $\beta$ - and $\gamma$-turns, as will be discussed below, although the general spectral features in this domain are similar between the two conformers. Both conformers hence give rise to a triplet between $\sim 490$ $540 \mathrm{~cm}^{-1}$ and to a doublet between $\sim 400-470 \mathrm{~cm}^{-1}$. Note that only the higher frequency band of the triplet $\left(518 \mathrm{~cm}^{-1}\right.$ for the $\gamma$-turn, $537 \mathrm{~cm}^{-1}$ for the $\beta$-turn) shows a substantial $19 \mathrm{~cm}^{-1}$ upshift when comparing the spectra of the two conformers. On the contrary, the doublet peaks are substantially shifted in position between the two conformers, with the two signatures being upshifted by +38 and $+29 \mathrm{~cm}^{-1}$ for the $\beta$ conformer with respect to the $\gamma$ conformer. It is also interesting to note that all peaks (triplet and doublet) in this domain are substantially more intense in the spectrum of the $\gamma$-turn conformer.

For the $\gamma$-turn, only a single intense peak is predicted in place of the experimental triplet, although much lower intensity peaks can be seen in the tail of the intense peak, while the triplet is correctly predicted for the $\beta$-turn. The doublet of the $\beta$-turn from the dynamical spectrum is blue-shifted from experiment. Interestingly, the subtle details of the $\gamma$-turn doublet are well predicted in the dynamical spectrum, up to the $475 \mathrm{~cm}^{-1}$ tail peak. We find that the triplet and doublet features are due to $\mathrm{N}-\mathrm{H}$ torsional/out-of-plane motions, whether $\mathrm{N}-\mathrm{H}$ belongs to the $\mathrm{NH}_{2}$ function (free and $\mathrm{H}$-bonded $\mathrm{N}-\mathrm{H}$ signatures in this domain) or to the amide backbone $\mathrm{N}-\mathrm{H}$. The triplet in the $\gamma$-turn arises from a combination of all $\mathrm{N}-\mathrm{H}$ signatures, merging into one single peak in the BOMD spectrum, in contrast to the experiment. The triplet in the $\beta$-turn is on the contrary solely due to the free $\mathrm{N}-\mathrm{H}$ group of the $\mathrm{NH}_{2}$ terminus of the peptide. For both $\beta$ - and $\gamma$-turns, the doublet clearly reflects the signature of the free $\mathrm{N}-\mathrm{H}$ of the $\mathrm{NH}_{2}$ function, while this signature is also overlapping with the $\mathrm{NH} \cdots \mathrm{O} \mathrm{H}$-bond signature for the $\beta$-turn conformer.

The doublet is thus directly ( $\beta$-turn)/indirectly ( $\gamma$-turn) related to the $\mathrm{C} 10 / \mathrm{C} 7$ hydrogen bond. It is clear from Fig. 6 where the evolution with time of the $\mathrm{O} \cdots \mathrm{H} \mathrm{H}$-bond of the C10 $\beta$-turn (blue line) and of the C7 $\gamma$-turn (red line) conformers is reported, that the average $\mathrm{H}$-bond length and the fluctuations around the average differ between the two conformers (same temperature for the two trajectories). While this H-bond is $2.01 \pm 0.08 \AA$ on average for the $\gamma$-turn conformer, it is $1.95 \pm 0.09 \AA$, on average for the $\beta$-turn conformer (see Table S2, ESI $\dagger$ ). This shows how the C10 ring of the $\beta$-turn is more tightly $\mathrm{H}$-bonded than the $\mathrm{C} 7 \mathrm{ring}$ of the $\gamma$-turn. This difference directly reflects the strength of the hydrogen bond, with the C7 $\gamma$-turn H-bond being weaker. With this strength difference in mind, one is therefore not surprised that the signature of the $\mathrm{C} 7 \mathrm{H}$-bond in the $\gamma$-turn conformer appears at lower frequencies, and that the $\mathrm{C} 10$ has a more direct signature in the doublet assignment than the C7. Experiments and dynamical spectra show that the $\mathrm{C} 10 \mathrm{H}$-bond signature is up-shifted from the C7 signature, roughly by $\sim 30-40 \mathrm{~cm}^{-1}$, providing a distinct signature of the $\gamma$-versus $\beta$-turn for the PhePro peptide.

One more comment about the weaker C7 H-bond ( $\gamma$-turn) with respect to the $\mathrm{C} 10$ one ( $\beta$-turn). It is well-known that GGA

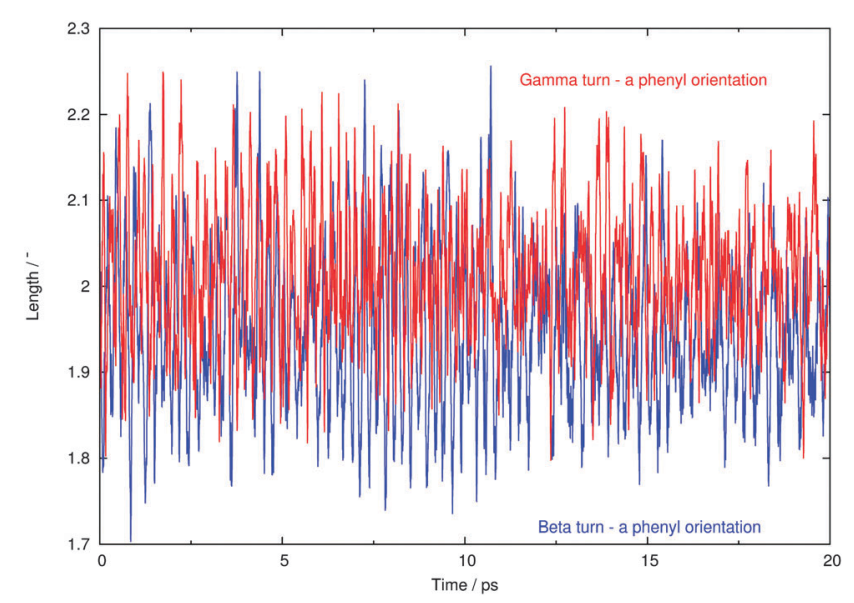

Fig. 6 Evolution with time of length of the $\mathrm{H} \ldots \mathrm{O} \mathrm{H}$-bond in the $\mathrm{C} 10$ $\beta$-turn (blue line) and in the C7 $\gamma$-turn (red line). This is plotted over 20 ps of the trajectories. 
functionals such as BLYP underestimate H-bond strengths, which consequently might allow too large amplitude motions of the hydrogen atoms involved in the hydrogen bond. This subsequently might lead to too large associated vibrational bands, which is indeed observed in the $450-480 \mathrm{~cm}^{-1}$ doublet in the dynamical spectrum of the $\beta$-conformer. These two bands also have lower intensities in the experimental spectra, showing that our representation presumably overestimates the $\mathrm{H}$-bond motions in the $\beta$-turn C10 interaction. This is also observed for the $\mathrm{C} 7$ interaction of the $\gamma$-turn, but to a lower extent.

To understand the spectral domain below $400 \mathrm{~cm}^{-1}$, we have used the ICDOS vibrational signatures (Internal Coordinate Density Of States) of backbone torsional motions, of the hydrogen bond and of the out-of-plane motion of $\mathrm{H}$ atoms that belong to the $\mathrm{NH}_{2}$ functional group. This is shown in Fig. 5 . First of all, the out-of-plane motion of the hydrogen atoms that belong to $\mathrm{NH}_{2}$ function do not seem to be relevant to explain the low-frequency vibrational features below $350 \mathrm{~cm}^{-1}$ since they do not show clear activity in this region. Only the peak at $378 \mathrm{~cm}^{-1}$ for the $\gamma$-turn is explained by the out-of-plane motion of the free hydrogen atom of the $\mathrm{NH}_{2}$ function. Second, as the $\mathrm{C} 7 / \mathrm{C} 10$ hydrogen bond leads to the folding of the peptide, we expect that both backbone motions and hydrogen bond motions are strongly coupled. This is indeed observed for the two conformers, as the hydrogen bond streching signature and dihedral backbone motion signatures share common features in the ICDOS spectra.

The spectra of the $\beta$ - and $\gamma$-turns do not appear to be strongly conformer selective in the $240-400 \mathrm{~cm}^{-1}$ range. There are 7 peaks in common between the two spectra, deviating in position by only $2-14 \mathrm{~cm}^{-1}$. Despite these similarities in the IR signatures, the assignments of the bands are surprisingly rather different. Although the intrinsic nature of the motions is similar, namely backbone motions, the coupling between these motions is different due to the two different backbone $3 \mathrm{D}$ folded structures in the $\gamma$ - and $\beta$-turns. For instance, the $306 / 308 \mathrm{~cm}^{-1}$ peak, respectively, for the $\gamma$-and $\beta$-turns, does not arise from the same motion. The former is related to the $\Phi_{\text {Pro }}$ torsional motion, while the latter comes from $\Phi_{\text {Phe }}$ torsion. Also, the two peaks located at $378 \mathrm{~cm}^{-1}$ for the $\gamma$-turn and $386 \mathrm{~cm}^{-1}$ for the $\beta$-turn are explained by different motions: the out-of-plane motion of the $\mathrm{NH}_{2}$ free hydrogen atom for the $\gamma$-turn, and coupled $\omega_{\text {Pro }}$ and $\Psi_{\text {Phe }}$ motions for the $\beta$-turn. Of special interest are the two peaks (experiment and dynamical spectra) recorded in the $250-300 \mathrm{~cm}^{-1}$ domain that carry $\gamma$ - versus $\beta$-turn selectivity. They, respectively, differ by +17 and $+14 \mathrm{~cm}^{-1}$ (from lower to higher frequency), going from $\gamma$ - to $\beta$-conformer spectra: these two peaks are due to the amide peptide backbone motions namely $\Phi_{\text {Pro }}$ (lower frequency) and $\Phi_{\text {Phe }}$ (higher frequency). These backbone motions are direct probes of the $\mathrm{C} 7 / \mathrm{C} 10$ folding of PhePro, thus providing conformer specific spectral signatures.

Although the $\mathrm{H}$-bond strength is clearly reflected by the peak position of the $\gamma$ - and $\beta$-turn conformers, the band pattern of the $\mathrm{C} 7 / \mathrm{C} 10 \mathrm{H}$-bond is very similar below $200 \mathrm{~cm}^{-1}$. The $\mathrm{H}$-bond signatures dominate the spectral assignments together with couplings to backbone torsions which are directly involved in the H-bond motions. There are four dominant bands related to this H-bond motion in the far-IR part of the spectra, respectively, located at $115 \mathrm{~cm}^{-1}, 140 \mathrm{~cm}^{-1}, 163 \mathrm{~cm}^{-1}$ and $174 \mathrm{~cm}^{-1}$ in the BOMD spectrum of the $\gamma$-turn and $111 \mathrm{~cm}^{-1}, 143 \mathrm{~cm}^{-1}$, $148 \mathrm{~cm}^{-1}$ and $175 \mathrm{~cm}^{-1}$ for the $\beta$-turn.

\section{Conclusions and outlook}

In this work, far-IR spectroscopy is shown to be a relevant tool for the characterization of peptide structures, since the observed far-IR features are a direct result from peptide backbone motions and hydrogen bond vibrations, and thereby directly reflect the secondary structure of peptides. The relationship between the delocalized backbone motions and the functional flexibility/ rigidity of peptides and proteins can thus be probed with this approach.

The experimental spectra reported here for two conformers of the Ac-Phe-Pro- $\mathrm{NH}_{2}$ peptide show very well-defined and wellresolved peaks from 800 down to $120 \mathrm{~cm}^{-1}$. To fully exploit the far-IR region and to retrieve the structural information hidden in this region, reliable calculations are key. The presented combination of conformation selective far-IR/UV double resonance spectroscopy with Born-Oppenheimer molecular dynamics simulations brings this synergy.

The present work is a follow-up on our previous initial demonstration $^{21}$ that such synergy was indeed able to differentiate the axial and equatorial forms of the $\gamma$-turn interaction in Ac-Phe-Gly- $\mathrm{NH}_{2}$, which was not possible with mid-IR spectroscopy and harmonic DFT calculations. ${ }^{22,23}$ Here, we have investigated two different turns in the conformation of Ac-Phe-Pro- $\mathrm{NH}_{2}$, i.e. $\gamma$ - (C7 H-bond interaction) and $\beta$-turns (C10 H-bond interaction), and have highlighted their specific far-IR signatures.

Clearly, the BOMD dynamical spectrum of the $\gamma$-turn conformation of Ac-Phe-Pro- $\mathrm{NH}_{2}$ provides a better agreement with the experiment than the dynamical spectrum of the $\beta$-turn. This is especially true below $350 \mathrm{~cm}^{-1}$ where the $\mathrm{H}$-bond signatures are present. One reason might be the use of the GGA/BLYP functional (although augmented here by D3 van der Waals interactions), known to underestimate the strength of $\mathrm{H}$-bonds, and thus allowing too large amplitude motions of the hydrogen atoms involved in H-bonds. One has also to keep in mind that nuclei quantum effects, especially of relevance to hydrogen atom motions, have not been taken into account in the present simulations. Such effects might help reduce some of the band-breadths, observed for $\mathrm{H}$-bonded motions of the $\beta$-turn conformer.

One main purpose of the combined experiment/BOMD spectra simulations was to provide conformer selective IR signatures of the $\gamma$-versus $\beta$-turns in the far-IR region. We have shown that the $400-550 \mathrm{~cm}^{-1}$ domain indeed provides such a distinction between the two conformers. This domain is predominantly due to $\mathrm{N}-\mathrm{H}$ motions, indirectly probing the $\mathrm{NH} \cdots \mathrm{O} \mathrm{H}$-bond motion. We have shown that there is a $29-38 \mathrm{~cm}^{-1}$ downshift in the positions of the associated bands for the weaker C7 
interaction in the $\gamma$-turn conformer with respect to the $\mathrm{C} 10$ interaction in the $\beta$-turn.

The $800-550 \mathrm{~cm}^{-1}$ spectral domain was shown not to be conformer selective as bands in both conformers have very similar positions and same assignments from out-of-plane $\mathrm{H}$ atom motions of the phenyl ring and pro residue, not sensitive to the $\gamma / \beta$ turns. Also the backbone torsional domain in the $250-400 \mathrm{~cm}^{-1}$ does not provide too much conformer selectivity. Only the $250-300 \mathrm{~cm}^{-1}$ peaks carry $\gamma$-versus $\beta$-turn selectivity, as they, respectively, differ by +17 and $+14 \mathrm{~cm}^{-1}$ (from lower to higher frequency) from the $\gamma$ - to the $\beta$-conformer. These two signatures are due to the amide peptide backbone motions, namely $\Phi_{\text {Pro }}$ (lower frequency) and $\Phi_{\text {Phe }}$ (higher frequency), which are direct probes of the $\mathrm{C} 7 / \mathrm{C} 10$ folding of PhePro. The supplementary H-bond signatures below $350 \mathrm{~cm}^{-1}$ have been shown to be of limited use for conformer selectivity.

Comparing the results of the $\gamma$-turn conformer of PhePro presented here with our previously published results on PheGly and PheAla $\gamma$-turns, ${ }^{21}$ one worth comment concerns the H-bond length and its signature within the three systems. This $\mathrm{H}$-bond length is substantially shorter in PhePro $(2.01 \AA$ for the $\gamma$-turn and $1.95 \AA$ for the $\beta$-turn) than in PheGly (2.14 $\AA$ ) and PheAla (2.12 $\AA$ ). As a result, this leads to higher frequencies associated with the H-bond stretching vibration for PhePro $\left(140-150 \mathrm{~cm}^{-1}\right.$ domain) than for PheGly and PheAla $\left(\sim 130 \mathrm{~cm}^{-1}\right.$ domain). Probably due to the stronger $\mathrm{H}$-bond strength, the $\mathrm{H}$-bond stretching vibration then couples more strongly to backbone torsional vibrations, as can be seen from the hydrogen bond stretching activity at higher frequencies $\left(e . g .380 \mathrm{~cm}^{-1}\right)$ in the $\gamma$-turn of PhePro. In PheGly and PheAla, those bands were not observed.

\section{Acknowledgements}

Computational resources were provided by support from HPC resources from GENCI-France (Grant 2012-2014 [072484]). This work is part of the research programme of the Stichting voor Fundamenteel Onderzoek der Materie (FOM), which is financially supported by the Nederlandse Organisatie voor Wetenschappelijk Onderzoek (NWO). Part of the FELIX laboratory is financed through the NWO BIG-programme. The calculations were sponsored by NWO Physical Sciences (EW) for the use of the supercomputer facilities at SurfSara. We also want to acknowledge Prof Michel Mons for providing the initial structure for the $\beta$-turn and for very fruitful discussions. We also acknowledge the very helpful comments from the reviewers, which significantly improved the quality of this paper.

\section{References}

1 D. Kennedy and C. Norman, Science, 2005, 309, 75.

2 M. S. de Vries and P. Hobza, Annu. Rev. Phys. Chem., 2007, 58, 585-612.

3 S. Jaeqx, J. Oomens and A. M. Rijs, Phys. Chem. Chem. Phys., 2013, 15, 16341-16352.
4 R. J. Plowright, E. Gloaguen and M. Mons, ChemPhysChem, 2011, 12, 1889-1899.

5 K. Schwing, H. Fricke, K. Bartl, J. Polkowska, T. Schrader and M. Gerhards, ChemPhysChem, 2012, 13, 1576-1582.

6 I. Hunig and K. Kleinermanns, Phys. Chem. Chem. Phys., 2004, 6, 2650-2658.

7 M. Cirtog, A. M. Rijs, Y. Loquais, V. Brenner, B. Tardivel, E. Gloaguen and M. Mons, J. Phys. Chem. Lett., 2012, 3, 3307-3311.

8 J. M. Bakker, L. M. Aleese, G. Meijer and G. von Helden, Phys. Rev. Lett., 2003, 91, 203003.

9 P. Carcabal, R. T. Kroemer, L. C. Snoek, J. P. Simons, J. M. Bakker, I. Compagnon, G. Meijer and G. V. Helden, Phys. Chem. Chem. Phys., 2004, 6, 4546-4552.

10 D. F. Plusquellic, K. Siegrist, E. J. Heilweil and O. Esenturk, ChemPhysChem, 2007, 8, 2412-2431.

11 R. Singh, D. K. George, J. B. Benedict, T. M. Korter and A. G. Markelz, J. Phys. Chem. A, 2012, 116, 10359-10364.

12 A. G. Markelz, IEEE J. Sel. Top. Quantum Electron., 2008, 14, 180-190.

13 G. Acbas, K. A. Niessen, E. H. Snell and A. G. Markelz, Nat. Commun., 2014, 36.

14 R. J. Falconer and A. G. Markelz, J. Infrared, Millimeter, Terahertz Waves, 2012, 33, 973-988.

15 K. Itoh and T. Shimanouchi, Biopolymers, 1967, 5, 921-930.

16 R. Langner and G. Zundel, J. Chem. Soc., Faraday Trans., 1995, 91, 3831-3838.

17 P. Godlewska, J. Jańczak, E. Kucharska, J. Hanuza, J. Lorenc, J. Michalski, L. Dymińska and Z. Wegliński, Spectrochim. Acta, Part A, 2014, 120, 304-313.

18 A. Abo-Riziq, L. Grace, B. Crews, M. P. Callahan, T. van Mourik and M. S. de Vries, J. Phys. Chem. A, 2011, 115, 6077-6087.

19 A. M. Rijs, M. Kabelac, A. Abo-Riziq, P. Hobza and M. S. de Vries, ChemPhysChem, 2011, 12, 1816-1821.

20 D. Oepts, A. F. G. van der Meer and P. W. van Amersfoort, Infrared Phys. Technol., 1995, 36, 297-308.

21 S. Jaeqx, J. Oomens, A. Cimas, M. P. Gaigeot and A. M. Rijs, Angew. Chem., Int. Ed., 2014, 53, 3663-3666.

22 W. Chin, F. Piuzzi, J. P. Dognon, I. Dimicoli and M. Mons, J. Chem. Phys., 2005, 123, 084301.

23 W. Chin, J. P. Dognon, F. Piuzzi, B. Tardivel, I. Dimicoli and M. Mons, J. Am. Chem. Soc., 2005, 127, 707-712.

24 V. Nibali and M. Havenith, J. Am. Chem. Soc., 2014, 136, 12800.

25 J. Sun, G. Niehues, H. Forbert, D. Decka, G. Schwaab, D. Marx and M. Havenith, J. Am. Chem. Soc., 2014, 136, 5031.

26 T. Luong, P. Verma, R. Mitra and M. Havenith, Biophys. J., 2011, 101, 925.

27 N. Heine and K. Asmis, Int. Rev. Phys. Chem., 2015, 34, 1.

28 J. Bowlan, D. J. Harding, J. Jalink, A. Kirilyuk, G. Meijer and A. Fielicke, J. Chem. Phys., 2013, 138, 031102.

29 W. Chin, M. Mons, J. P. Dognon, B. Piuzzi, F. Tardivel and I. Dimicoli, Phys. Chem. Chem. Phys., 2004, 6, 2700-2709.

30 A. M. Rijs and J. Oomens, Top. Curr. Chem., 2015, 364, $1-42$. 
31 A. M. Rijs and J. Oomens, IR Spectroscopic Techniques to Study Isolated Biomolecules, Springer International Publishing, 2015, vol. 364, pp. 1-42.

32 M. V. Johnston, Trends Anal. Chem., 1984, 3, 58-61.

33 J. VandeVondele, M. Krack, F. Mohamed, M. Parrinello, T. Chassaing and J. Hutter, Comput. Phys. Commun., 2005, 167, 103.

34 The CP2K developers group, http://www.cp2k.org/, 2013.

35 M. Krack, Theor. Chem. Acc., 2005, 114, 145.

36 S. Goedecker, M. Teter and J. Hutter, Phys. Rev. B: Condens. Matter Mater. Phys., 1996, 54, 1703.

37 C. Hartwigsen, S. Goedecker and J. Hutter, Phys. Rev. B: Condens. Matter Mater. Phys., 1998, 58, 3641.

38 A. Becke, Phys. Rev. A: At., Mol., Opt. Phys., 1988, 38, 3098.

39 C. Lee, W. Yang and R. G. Parr, Phys. Rev. B: Condens. Matter Mater. Phys., 1988, 37, 785.

40 S. Grimme, J. Antony, S. Ehrlich and H. Krieg, J. Chem. Phys., 2010, 132, 154104.

41 D. McQuarrie, Statistical Mechanics, Harper-Collins Publishers, New York, 1976.

42 R. Kubo, M. Toda and N. Hashitsume, Statistical Physics, Springer Verlag, 2nd edn, 1991, vol. II.

43 M. P. Gaigeot and M. Sprik, J. Phys. Chem. B, 2003, 107, 10344.

44 R. Iftimie and M. E. Tuckerman, J. Chem. Phys., 2005, 122, 214508.

45 H. Ahlborn, B. Space and P. B. Moore, J. Chem. Phys., 2000, 112, 8083.

46 J. Borysow, M. Moraldi and L. Frommhold, Mol. Phys., 1985, 56, 913.

47 R. Ramirez, T. Lopez-Ciudad, P. Kumar and D. Marx, J. Chem. Phys., 2004, 121, 3973.
48 C. P. Lawrence and J. L. Skinner, Proc. Natl. Acad. Sci. U. S. A., 2005, 102, 6720.

49 H. Kim and P. J. Rossky, J. Chem. Phys., 2006, 125, 074107.

50 M. P. Gaigeot, Phys. Chem. Chem. Phys., 2010, 12, 3336.

51 C. Marinica, G. Grégoire, C. Desfrançois, J. P. Schermann, D. Borgis and M. P. Gaigeot, J. Phys. Chem. A, 2006, 110, 8802 .

52 A. Cimas, T. D. Vaden, T. S. J. A. de Boer, L. C. Snoek and M. P. Gaigeot, J. Chem. Theory Comput., 2009, 5, 1068.

53 A. Cimas, P. Maitre, G. Ohanessian and M. P. Gaigeot, J. Chem. Theory Comput., 2009, 5, 2388.

54 A. Sediki, L. C. Snoek and M.-P. Gaigeot, Int. J. Mass Spectrom., 2011, 308, 281.

55 J. P. Beck, M.-P. Gaigeot and J. M. Lisy, Phys. Chem. Chem. Phys., 2013, 15, 16736.

56 M. P. Gaigeot, R. Vuilleumier, M. Sprik and D. Borgis, J. Chem. Theory Comput., 2005, 1, 772.

57 J. W. Handgraaf, E. J. Meijer and M. P. Gaigeot, J. Chem. Phys., 2004, 121, 10111-10119.

58 M. P. Gaigeot, Phys. Chem. Chem. Phys., 2010, 12, 10198.

59 D. Bovi, A. Mezzetti, R. Vuilleumier, M.-P. Gaigeot, B. Chazallon, R. Spezia and L. Guidoni, Phys. Chem. Chem. Phys., 2011, 13, 20954.

60 M. Sulpizi, M. Gaigeot and M. Sprik, J. Chem. Theory Comput., 2012, 8, 1037.

61 M.-P. Gaigeot, M. Sprik and M. Sulpizi, J. Phys.: Condens. Matter, 2012, 24, 124106.

62 M. Sulpizi, M. Salanne, M. Sprik and M. Gaigeot, J. Phys. Chem. Lett., 2013, 4, 83.

63 B. Yan, S. Jaeqx, W. J. van der Zande and A. M. Rijs, Phys. Chem. Chem. Phys., 2014, 16, 10770-10778. 